\title{
Passeurs de frontières
}

\section{Erhard Taverna}

Dr med., membre de la rédaction

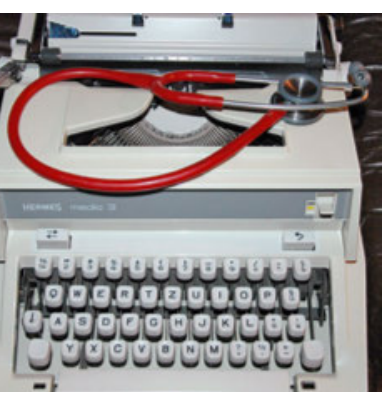

Quitter son domaine, avoir une double appartenance, par exemple en écrivant. La littérature n'est pas le privilège d'une profession. Les passeurs de frontières sont aussi ceux qui amènent une particularité en traduisant leur jargon dans une autre langue, avec créativité. Comme ces célèbres physiciens, Stephen Hawking, Ben Moore ou Lisa Randall, qui nous expliquent l'univers, à nous profanes, grâce à des ouvrages pratiques pleins d'imagination. D'autres scientifiques prennent aussi le temps et ont le talent de transmettre leurs connaissances à un vaste public.

\section{Les passeurs de frontières sont aussi ceux qui amènent une particularité en traduisant leur jargon dans une autre langue, avec créativité.}

On peut citer, pour illustrer cette symbiose entre différents dons, le chimiste Carl Djerassi (1923-2015), maintes fois récompensé, pour la première synthèse d'un contraceptif oral, mais aussi pour des nouvelles, romans et pièces de théâtre. An Immaculate Miconception traite du thème de sa vie, la séparation entre sexe et fertilité, à travers le personnage d'une scientifique testant une nouvelle méthode d'autofécondation. Moins connu, Jean Lindenmann (1924-2015), directeur de l'institut d'immunologie et de virologie de l'université de Zurich et qui a découvert la cytokine Interféron, a traité un problème similaire par la littérature. En s'inspirant des Affinités électives de Goethe, il a rédigé une histoire d'inceste génétique, Die Leihverwandtschaften, un drame sur les conséquences d'un 'viol en éprouvette'. D'une manière exceptionnelle, le neurologue britannique Oliver Sacks (19332015) a transformé des tableaux cliniques complexes en drames existentiels célèbres dans le monde entier. Tous les trois ont réfléchi aux problèmes éthiques de leur métier en dehors de la littérature spécialisée, en les transposant sous forme littéraire.

Beaucoup de médecins ont écrit des œuvres importantes. Dans le domaine germanique, Schnitzler, Benn et Döblin sont les plus cités. Chez certains, l'expérience médicale est le thème principal, d'autres choisissent une manière de raconter et des questions n'évoquant aucun lien avec leur travail. Comme Eduard Renner (1891-1952), médecin de Suisse centrale. Le livre Goldener Ring über Uri, une interprétation de la vie des alpagistes, n'aurait jamais vu le jour sans son travail en cabinet. Dans ses romans Wüthrich et Schizogorsk, le radiologue et psychiatre Walter Vogt (1927-1988) décrit un univers cauchemardesque, sous la forme de la visite d'un professeur de médecine ou de l'histoire d'une patiente ayant des conséquences criminalistiques. Les nombreuses consultations de Lukas Fierz (1941), ancien membre du Conseil national, ont donné Reportagen aus der heilen Schweiz - Begegnungen mit dem Leibhaftigen. Nombreux sont les collègues qui transforment avec art leur quotidien professionnel en histoires policières, ce qui donne des livres passionnants, souvent burlesques et critiques sur la société. Nous en avons présenté quelques-uns dans la critique littéraire du Bulletin des médecins suisses.

\section{Nombreux sont les collègues qui transforment} avec art leur quotidien professionnel en histoires policières.

Certains journaux intimes, essais, chroniques, voire courriers de lecteurs peuvent aussi être inventifs. Ces formes d'expression relèvent également d'un double don, la compétence dans son métier principal et une aptitude dans un autre domaine. En 1968, l'Union Mondiale des Médecins Ecrivains UMEM a été créée à Lucerne. Beaucoup recherchent le contact au sein des syndicats du pays, d'autres préfèrent trouver l'inspiration à l'extérieur. Anton Tchekhov évoquait ainsi cette volupté: «La médecine est ma femme légitime, la littérature, ma maîtresse. Quand l'une m'ennuie, je vais passer ma nuit avec l'autre.» Les passeurs de frontières ont souvent une double appartenance, quelle que soit l'activité de prédilection de ces médecins, faire de la musique, de la sculpture, tourner des films ou animer des émissions de télévision. Une seconde corde à leur arc, pas toujours nécessaire, mais toujours sympathique.

\section{Crédit photo \\ Erhard Tavern}

